eCommons@AKU

April 2008

\title{
Factors influencing in-hospital length of stay and mortality in cancer patients suffering from febrile neutropenia
}

\author{
Amar Lal \\ Aga Khan University \\ Yasmin Bhurgri \\ Aga Khan University \\ Nida Rizvi \\ Aga Khan University \\ Mohni Virwani \\ Aga Khan University \\ Rasheed Uddin Memon \\ Aga Khan University \\ See next page for additional authors
}

Follow this and additional works at: https://ecommons.aku.edu/

pakistan_fhs_mc_med_haematol_oncol

Part of the Hematology Commons, and the Oncology Commons

\section{Recommended Citation}

Lal, A., Bhurgri, Y., Rizvi, N., Virwani, M., Memon, R., Saeed, W., Sardar, M., Kumar, P., Shaikh, A., Adil, S., Masood, N., Khurshid, M. (2008). Factors influencing in-hospital length of stay and mortality in cancer patients suffering from febrile neutropenia. Asian Pacific Journal of Cancer Prevention: Apjcp, 9(2), 303-8.

Available at: https://ecommons.aku.edu/pakistan_fhs_mc_med_haematol_oncol/3 


\section{Authors}

Amar Lal, Yasmin Bhurgri, Nida Rizvi, Mohni Virwani, Rasheed Uddin Memon, Wajeeha Saeed, Muhammad Rizwan Sardar, Pawan Kumar, Asim Jamal Shaikh, Salman Adil, Nehal Masood, and Mohammad Khurshid 


\title{
RESEARCH COMMUNICATION
}

\section{Factors Influencing In-hospital Length of Stay and Mortality in Cancer Patients Suffering from Febrile Neutropenia}

\author{
Amar Lal', Yasmin Bhurgri²*, Nida Rizvi', Mohni Virwani' ${ }^{1}$, Rasheed Uddin \\ Memon', Wajeeha Saeed ${ }^{1}$, MuhammadRizwan Sardar', Pawan Kumar'1, Asim \\ Jamal Shaikh ${ }^{1}$, Salman Adil ${ }^{1}$, Nehal Masood ${ }^{1}$, Mohammed Khurshed ${ }^{1}$
}

\begin{abstract}
Introduction: Febrile neutropenia (FN) is a major complication of chemotherapy, costly in terms of morbidity, mortality and associated financial expenditure. The present study was conducted with the goal of highlighting FN as a serious problem in Pakistan, with the longer term objective of improved cancer survival, reduction in length of stay (LOS) in hospital, morbidity, mortality and costs in our existing developing country scenario. Methods: A cross-sectional descriptive study was conducted on patients, $\geq 18$ years, admitted with FN as a consequence of chemotherapy at a referral hospital in Karachi from 1st September 2006 to 30th April 2007. Results: A total of 80 patients [43 (53.8\%) males and $37(46.2 \%)$ females] were selected. The mean age was 47.4 (SD \pm 16.6 ; range 18-79) years. Sixty eight patients $(86 \%)$ were $\leq 65$ years, $50 \%$ were $\leq 50$ years. Overall, inhospital mortality was $11 \% ; 4 \%$ for patients on granulocyte colony stimulating factor (G-CSF) prophylaxis as against $20 \%$ for those without. The cause of death was either pneumonia or septic shock. Mean LOS was 7.53 (SD \pm 3.8 ; range 2-17) days. Hematological malignancies, older age, severity of dehydration, pneumonia and culture positivity were significantly associated with LOS and death. Those above 50 years of age were 1.5 times as likely to be hospitalized longer and > three times as likely to die. Bacteremia conferred a 5-fold and pneumonia an 8-fold increase in the risk of death. Conclusion: The results of this study indicate that age, vital instability, dehydration, high creatinine, culture positivity and hematological malignancies are high risk factors in chemotherapy induced FN. Identification of FN risk factors with poor outcomes may help in devising protocols for modified dosage or including GCFs initially. This may help reduce the cost of cancer care as well as mortality and morbidity. Prospective studies of FN in multiple centers in Pakistan may be beneficial in evaluating these risk factors further.
\end{abstract}

Key Words: Febrile neutropenia - cancer patients - in-hospital stay - mortality - Pakistan

Asian Pacific J Cancer Prev, 9, 303-308

\section{Introduction}

Febrile neutropenia $(\mathrm{FN})$ is a condition marked by fever developing in patients suffering from neutropenia. The fever is caused by infection in $50 \%$ of cases and bacteremia may be present in as many as $20 \%$ of the patients with an absolute neutrophil count under 1,000 cells/ mcL (mm3) (Hughes et al., 2002). According to the Infectious Disease Society of America (IDSA) and the US Food and Drug Administration (FDA), a single temperature $\geq 38.3^{\circ} \mathrm{C}(1010 \mathrm{~F})$ orally or a temperature of $38.0^{\circ} \mathrm{C}(100.40 \mathrm{~F})$ over 1 hour in the absence of an obvious cause, is defined as fever and $\leq 500$ neutrophils/mcL or $\leq 1,000$ neutrophils/mcL and a predicted decline to $500 /$ mcL over the next 48 hours is defined as neutropenia (Hughes et al., 2002).

FN is causally associated with myelosuppressive chemotherapy in adults. Affected patients are empirically treated with antibiotics until the neutrophil count has recovered. Guidelines issued in 2002 by IDSA recommend the use of combinations of antibiotics in specific settings; mild low-risk cases may be treated with a combination of oral co-amoxiclav and ciprofloxacin, while more severe cases require cefalosporins with activity against Pseudomonas aeruginosa (e.g. ceftazidime), or carbapenems (imipenem or meropenem) (Hughes et al., 2002). Excluding infection, the other causes of fever in cancer patients are, tumor-associated factors, allergic or hypersensitivity reaction to drugs especially amphotericin, biologics and some cytotoxic agents and allergic or hypersensitivity reactions to blood component therapies (Hughes et al., 2002).

$\mathrm{FN}$ is costly in terms of morbidity, mortality and associated financial expenditure. A number of different 
models based on the characteristics demonstrated by patients at onset of febrile neutropenia have been designed to enable clinicians to assign patients to high or low-risk groups thus attempting to identify patients with the greatest chance of recovering without serious medical complication and also those who might encounter difficult sequels (Talcott et al., 1988).

The Multinational Association of Supportive Care in Cancer (MASCC) risk-index score identifies low-risk patients (score $\geq 21$ points) at the onset of FN. These patients are at a risk of developing serious complications of FN (including death, intensive care unit admission, confusion, cardiac, renal and respiratory failure, hypotension and hemorrhage (Klastersky J et al, 2000). It also helps to establish the current guidelines for the treatment of FN by the IDSA as well as the recommendations for the use of hematopoietic colonystimulating factors (CSFs) by the American Society of Clinical Oncology (ASCO) (Klastersky et al., 2000; Basu et al., 2005).

The main objective of this study was to highlight FN as a serious complication of chemotherapy, identify risk factors, appreciate established FN guidelines, use them for prophylaxis and to predict outcomes especially in our setup in terms primarily of length of hospital stay (LOS) and mortality associated with FN and thus improve patient survival.

\section{Materials and Methods}

This study was designed as a cross-sectional descriptive study. All patients, 18 years and above, who had a biopsy proven cancer, been exposed to chemotherapeutic agent/s, found febrile $\left(>38^{\circ} \mathrm{C}\right)$ and neutropenic (below 500 cells $/ \mu \mathrm{L}$ ) were included in the study. Patients in the setting of immunodeficiency status or neutropenia associated with syndromes that are not associated with a high risk of bacterial infection (e.g. chronic benign neutropenia) were excluded from the study.

The major outcomes of interest were length of stay (LOS) in hospital and mortality. The major independent variables of interest were age, sex, histopathologic diagnosis, associated complications, co-morbid conditions, clinical presentation including severity of dehydration, oral mucositis, hematological and biochemical changes including severity of neutropenia in cancer patients with chemotherapeutic induced febrile neutropenia.

Patients of FN who were admitted from 1st September 2006 to 30th April 2007 at the Section of Medical Oncology and Hematology, Department of Medicine, the Aga Khan University Hospital, Karachi were recruited for the study, after obtaining written informed consent. At admission, all eligible patients with FN had a detailed history and examination by the admitting residents. Blood was drawn for Complete Blood Count (CBC), creatinine, serum electrolytes, Liver Function Tests (LFTs), blood culture and sensitivity $(\mathrm{C} / \mathrm{S})$ from the peripheral veins and also from the central venous access devices (Port-A-cath, Hickman Line, Central Venous Line).

Urine culture and chest X-rays were taken and in selected patients ultrasonography was done to identify the source of infection and also to identify the disease severity. Empirical intravenous antibiotics were started after drawing blood and urine cultures. Every patient was followed throughout the length of hospital stay. Data collection forms were used to collect data regarding demographics, laboratory parameters, cancer type, comorbid medical illnesses and complications and LOS along with outcome (discharged, died, left against medical advice).

The primary outcomes of this analysis were LOS and mortality in the hospital, which served as markers for the severity of illness. Univariate association between each outcome variable (length of stay and mortality) and each of the independent variables was then evaluated. Length of stay was used as a dichotomous variable ( $\leq 5$ days and $>5$ days) for the purpose of the initial univariate analyses. The cut-off point of 5 days for the purpose of the analysis was determined before the data analysis and was based on the clinical expectation that a patient who was admitted for less than 5 days most likely represented one who had an uncomplicated disease-course in the hospital.

\section{Results}

A total of 80 patients [43 (53.8\%) males and 37 (46.2\%) females] were selected for the study using the criteria described in the methodology. The mean age was 47.4 (95\%CI 43.66, 51.06; SD 16.6; range 18-79) years. Sixty eight patients $(86 \%)$ were $\leq 65$ years and $50 \%$ were $\leq 50$ years of age. The majority of the patients hailed from Karachi $(65,81.3 \%)$, however $14(17.5 \%)$ were residents of other regions of Pakistan and $1(1.2 \%)$ patient came from Kabul, Afghanistan.

The topographic distribution of tumors according to the primary site was - solid tumors (29 cases, $37.5 \%)$ and hematological malignancies (51 cases, $63.8 \%$ ). The distribution of solid cancers was breast cancer (17 cases, $58.6 \%$ ), ovarian cancer (4 cases, $13.8 \%$ ), gastric cancer, lung cancer, and Ewing's sarcoma (2 cases, 6.9\%) each and uterine cancer and immature teratoma (1 case, $3.45 \%$ ) each. The sub-distribution of hematological malignancies was non-Hodgkin's lymphoma, NHL (30 cases, 58.8\%),

\section{Table 1. Frequencies of Predictor Variables}

\begin{tabular}{|c|c|c|}
\hline & Frequency & Percentage \\
\hline \multicolumn{3}{|l|}{ Temperature } \\
\hline$<38^{\circ} \mathrm{C} / \geq 38^{\circ} \mathrm{C}$ & $16 / 64$ & $20.0 / 80.0$ \\
\hline \multicolumn{3}{|l|}{ Pulse rate } \\
\hline$\leq 80 / \geq 80$ & $14 / 66$ & $17.7 / 82.3$ \\
\hline \multicolumn{3}{|l|}{ Hemoglobin } \\
\hline$<10 / 10 \&$ above & $34 / 46$ & $21.5 / 58.5$ \\
\hline \multicolumn{3}{|l|}{ Platelet } \\
\hline$<50 / 50 \&$ above & $20 / 60$ & $25.0 / 75.0$ \\
\hline \multicolumn{3}{|l|}{ Platelet?? } \\
\hline$<20 / 20 \&$ above & $12 / 68$ & $15.0 / 85.0$ \\
\hline \multicolumn{3}{|l|}{ ANC } \\
\hline$<100 / 100$ and above & $64 / 16$ & $80.0 / 20.0$ \\
\hline \multicolumn{3}{|l|}{ S. Creatinine } \\
\hline$<1 / 1$ and above & $71 / 9$ & $88.9 / 11.1$ \\
\hline Total & 80 & 100.0 \\
\hline
\end{tabular}


Table 2. Bivariate Association between Each Predictor Variable and Length of Stay

\begin{tabular}{|c|c|c|c|c|}
\hline Factor & Mean (SD) & Difference & $95 \%$ C.I. & $\mathrm{p}$-value \\
\hline \multicolumn{5}{|l|}{ Solid/Liquid } \\
\hline Solid & $6.0(3.5)$ & & & \\
\hline Liquid & $8.5(3.8)$ & -2.5 & $-4.2,-0.8$ & 0.004 \\
\hline \multicolumn{5}{|l|}{ Co-Morbidity } \\
\hline Yes & $7.3(3.9)$ & & & \\
\hline No & $7.6(3.8)$ & 0.3 & $-1.5,2.2$ & 0.702 \\
\hline \multicolumn{5}{|c|}{ Taken Prophylactic } \\
\hline Yes & $7.0(4.0)$ & & & \\
\hline No & $7.9(3.7)$ & -0.9 & $-2.7,0.8$ & 0.283 \\
\hline \multicolumn{5}{|c|}{ Severely abnormal ANC } \\
\hline Yes & $8.8(4.1)$ & & & \\
\hline No & $7.2(3.7)$ & -1.6 & $-3.7,0.4$ & 0.116 \\
\hline \multicolumn{5}{|c|}{ Severity of Dehydration } \\
\hline Normal-Mild & d $\quad 5.2(2.2)$ & & & \\
\hline Mod-Severe & $11.4(2.7)$ & -6.2 & $-7.3,-5.0$ & $<0.001$ \\
\hline \multicolumn{5}{|l|}{ ER Creatinine } \\
\hline$<1$ & $7.1(4.0)$ & & & \\
\hline 1 or more & $8.9(3.4)$ & -1.8 & $-3.7,0.2$ & 0.079 \\
\hline \multicolumn{5}{|l|}{ Blood Culture } \\
\hline Positive & $6.3(3.4)$ & & & \\
\hline Negative & $10.0(3.5)$ & -3.7 & $-5.3,-2.1$ & $<0.001$ \\
\hline \multicolumn{5}{|c|}{ Pneumonia on Chest X-ray } \\
\hline Yes & $9.7(4.0)$ & & & \\
\hline No & $6.8(3.5)$ & -2.9 & $-4.7,-0.96$ & 0.004 \\
\hline \multicolumn{5}{|l|}{$\mathrm{Hb}$ Level in ER } \\
\hline$<10$ & $8.6(3.6)$ & & & \\
\hline $10 \&$ above & $6.8(3.8)$ & 1.8 & $0.06,3.5$ & 0.043 \\
\hline \multicolumn{5}{|c|}{ Platelet Level in ER } \\
\hline$<50$ & $8.5(4.1)$ & & & \\
\hline $50 \&$ above & $7.2(3.7)$ & 1.3 & $-0.7,3.3$ & 0.191 \\
\hline
\end{tabular}

Table 3. Bivariate Association between Each Predictor Variable and Mortality

\begin{tabular}{|c|c|c|c|c|}
\hline & Yes* & No* & Odds Ratio (95\% C.I.) & $\mathrm{p}$-value \\
\hline \multicolumn{4}{|c|}{ Solid/Liquid Tumor } & 0.263 \\
\hline Solid & 2 & 29 & 1 & \\
\hline Liquid & 7 & 42 & $2.42(0.47,12.5)$ & \\
\hline \multicolumn{4}{|c|}{ Severely abnormal ANC } & 0.370 \\
\hline Yes & 3 & 14 & $0.49(0.11,2.21)$ & \\
\hline No & 6 & 57 & 1 & \\
\hline \multicolumn{4}{|c|}{ Severity of Dehydration } & 0.059 \\
\hline Normal/Mild & d 3 & 47 & 1 & \\
\hline Mod/Severe & 6 & 24 & $3.92(0.90,17.04)$ & \\
\hline \multicolumn{4}{|c|}{ ER Creatinine } & 0.298 \\
\hline$<1$ & 4 & 54 & 1 & \\
\hline 1 or more & 3 & 17 & $2.38(0.48,11.7)$ & \\
\hline \multicolumn{4}{|l|}{ Blood Culture } & 0.032 \\
\hline Positive & 6 & 21 & $4.76(1.09,20.9)$ & \\
\hline Negative & 3 & 50 & 1 & \\
\hline \multicolumn{4}{|c|}{ Pneumonia on Chest X-ray } & 0.005 \\
\hline Yes & 6 & 14 & $8.14(1.81,36.7)$ & \\
\hline No & 3 & 57 & 1 & \\
\hline \multicolumn{4}{|c|}{$\mathrm{Hb}$ Level in Emergency/OPD } & 0.279 \\
\hline$<10$ & 5 & 26 & $2.16(0.53,8.78)$ & \\
\hline $10 \&$ above & 4 & 46 & 1 & \\
\hline \multicolumn{4}{|c|}{ Platelet Level in Emergency/OPD } & 0.551 \\
\hline$<50$ & 3 & 17 & $1.59(0.36,7.04)$ & \\
\hline $50 \&$ above & 6 & 54 & 1 & \\
\hline
\end{tabular}

*Death within one month 1 - reference category acute myeloid leukemia (AML) and acute lymphoid leukemia (ALL) 7 (13.7\%) cases each, multiple myeloma (MM) 3 (5.88\%) cases, Hodgkin's lymphoma (HD), chronic lymphoid leukemia (CLL), chronic myeloid leukemia (CML) and follicular lymphoma (FL) 1 (1.96\%) case each. Metastatic disease was seen in $33(41.3 \%)$ cases whereas $47(58.8 \%)$ patients had localized disease.

Twenty five $(31.5 \%)$ patients had one or more comorbids. The co-morbid conditions were diabetes mellitus (DM), chronic renal failure (CRF), and DM with CRF (9 cases, $11.3 \%$ ) each, COPD and hypothyroidism 1 (1.3\%) case each. On admission, 30 (37.5\%) subjects had severe dehydration, 10 (12.5\%) had abnormal creatinine levels, $17(21.3 \%)$ had severely abnormal ANC, 31 (38.8\%) had a low hemoglobin and $20(25.0 \%)$ had a low platelet count. There were $21(26.3 \%)$ patients with a positive blood culture and $25(20.0 \%)$ subjects with pneumonia (Table $1)$.

The mean LOS was 7.53 (95\%CI 6.67, 8.83; SD 3.8; range 2-17) days. The study sample consisted of 29 (36.3\%) patients with a length of stay of 5 days or less and $31(63.7 \%)$ patients with length of stay longer than 5 days. The mean and median length of stay for the patients admitted for 5 days or less were the same ( 3 days), with a range of 1 to 5 days. The mean and median length of stay for the patients admitted for longer than 5 days were 19 days and 12 days, respectively, with a range of 6 to 359 days.

Patients had been febrile for 1.48 (95\%CI 1.31, 1.64; SD .75; range 1-3) days at the time of admission and neutropenic for 1.84 (95\%CI 1.67, 2.01; SD .77; range 1$3)$ days. An average of 10.28 (95\%CI 8.14, 12.41; SD 9.6; range 2-87) days had elapsed since the patients started chemotherapy. An average of 5.26 (95\%CI 3.62, 6.89; SD 7.3; range 1-36) months had elapsed since the patients had been diagnosed with cancer. Thirty five patients (43.8\%) had taken prophylactic antibiotics within the past 7 days before developing FN whereas $45(56.3 \%)$ of the patients were not on antibiotic cover. Forty five (56.3\%) patients were on G-CSF prophylaxis.

As seen in Table 2, patients with a hematological malignancy were found to be at a greater risk of being hospitalized for longer than 5 days as compared with subjects with solid cancer. Similarly patients with severe dehydration, low hemoglobin, positive blood culture and pneumonia were also significantly more likely to have a longer length of stay as compared with subjects without these associated conditions (Table 2).

Nine patients died in the study, leading to a mortality of $11.3 \%$. Four percent of the patients on G-CSF prophylaxis died of complications associated with FN as against $20 \%$ of those without G-CSF cover. The cause of death was either pneumonia or septic shock. Patients with hematological malignancies, those who were severely dehydrated those with positive blood cultures and pneumonia had an increased risk of death, as seen in Table 3. After controlling for cancer type and complications the elderly group were almost 1.5 times as likely to be hospitalized longer and more than three times as likely to die as the younger group below 50 years of age. Vital instability, severity of dehydration and bacteremias were 
significantly associated with both longer LOS and mortality. A diagnosis of bacteremia conferred a 5-fold increase in the risk of death. Pneumonia was associated with an eight-fold increase in the risk of death.

\section{Discussion}

Poor cancer survival has been lamented in Karachi and largely in Pakistan, whereby a large number of cancer patients die within the first year of diagnosis (Bhurgri et al., 2004). One of the causes of early mortality is FN, a life-threatening infection, associated with lengthy hospitalization and high morbidity and medical costs. In addition, neutropenia is the primary cause of dose reductions and dose delays, limiting the delivery of the chemotherapy at full dose, on schedule, thus further compromising long-term survival in patients with potentially curable malignancies. The overall goal of this study was to better understand factors associated with outcomes of FN. The objective being improved cancer survival, reduction in LOS, morbidity, mortality and if possible costs in our existing developing country scenario.

In the present study FN emerges as a high mortality complication of myelosuppressive chemotherapy. The elderly, those with hematological malignancies, the severely dehydrated patients, those with positive blood cultures and pneumonia were at an increased risk of longer LOS and death. Bacteremia conferred a 5-fold increase and pneumonia an eight-fold increase in the risk of death. These findings collaborate with other published studies. In comparison to solid tumors, a diagnosis of hematological malignancies particularly AML and NHL was associated with a three-fold increased risk of longer LOS as well as mortality. A diagnosis of breast cancer and ovarian cancer was associated with a significant reduction in the risk of both. The severity of neutropenia and abnormal creatinine were significantly associated with both longer LOS and mortality.

In studies of adults with febrile neutropenia, older age has emerged as a significant risk factor for poor outcomes (Morrison et al., 2001). Other factors associated with increased mortality, LOS and cost in hospitalized adult cancer patients with FN include patient characteristics, type of malignancy, co-morbidities, and infectious complications. Recognition of these factors may be useful in identifying patients at increased risk of serious medical complications and mortality for more aggressive supportive care measures (Kuerer et al., 2006). Many recent studies have documented that the greatest risk of neutropenia and its complications is in the first cycle of chemotherapy, whence $>50 \%$ of the first episodes of neutropenia and FN occur (Ozer, 2006; Ray-Coquard et al., 2006). FN also occurs more commonly during the first few days of each subsequent cycle of chemotherapy. Thus a better initial predictive approach may allow better therapeutic decisions for these patients, with an eventual impact on reducing mortality (Vincenzino, 1995).

There is a trend towards prophylactic, empirical use of antibiotics including Ceftriaxone and Amikacin for febrile neutropenia, oral Itraconazole for antifungal prophylaxis and oral acyclovir for antiviral prophylaxis
(Shamsi et al., 2004). There is also an increasing trend of gram negative organisms developing resistance to commonly used antibiotics. Gram positive bacteria are also showing emerging resistance to vancomycin (Khan et al., 2004). In recent years, there has been increasing interest in stratifying patients with $\mathrm{FN}$ into risk categories to explore various therapeutic strategies (Talcott et al., 1988; Klastersky et al., 2000; Ozer et al., 2000; Basu et al., 2005). Granulocyte colony-stimulating factors (GCSFs) have been shown to help prevent febrile neutropenia in certain subgroups of cancer patients undergoing chemotherapy, but their role in treating febrile neutropenia is controversial (Rocio García-Carbonero et al, 2001). The use of pegfilgrastim from the first cycle has been shown to reduce the incidence of febrile neutropenia in patients receiving moderately myelosuppressive chemotherapy and may be particularly beneficial in elderly patients (Weber, 1993; Balducci and Lyman, 2001; Chrischilles et al., 2002; Bennett and Schumock, 2003; Lyman et al., 2003; Repetto et al., 2003; Lyman, 2005; Caggiano et al., 2005).

Management of FN remains a costly challenge. Chemotherapy-related hematological adverse effects globally result in a substantial economic burden on patients, payers, caregivers and society in general (Liou et al., 2007). In Pakistan, a country of low to medium economic resource, which is not a welfare state, there is a substantial economic burden on cancer patients and caregivers especially the elderly. Employers do not bear the responsibility of treatment costs, moreover the retirement age is 60 years, there are little health related retirement benefits and the concept of health insurance is nonexistent. In the past, there have been attempts to identify patients who could be managed in an outpatient basis to save the cost incurred during inpatient management. Malik et al compared out-patient treatment with an oral antibiotic (ofloxacin) to in-patient treatment with this same antibiotic in patients experiencing neutropenia of short duration. A considerable proportion $(21 \%)$ of their subjects receiving oral antibiotic treatment as out-patients required hospitalization and this group had a $4 \%$ mortality rate, raising concerns about the safety of this approach (Malik et al., 1995).

Delayed hospitalization remains an associated problem in the management of FN either due to avoidance of costs or a failure to recognize $\mathrm{FN}$ as a serious complication. In our study conducted at a major referral centre, the patients had been febrile and neutropenic for approximately 2 days at the time of admission and had been on chemotherapy for atleast 10 days. The delay in referral compounds the problem of FN management, as patients report in a critical state, the mortality in our study being $11.25 \%$. In the present study, those subjects who were on G-CSF prophylactic cover had a lower mortality from FN. Most studies support the finding that adding G-CSF to antibiotic therapy shortens the duration of neutropenia, reduces the duration of antibiotic therapy and hospitalization, and decreases hospital costs in patients with high-risk febrile neutropenia. The therapeutic role of G-CSF in LOS and mortality could not be studied as all patients were given 
G-CSFs.

G-CSF treatment may offer substantial potential savings for hospitalized patients with established neutropenia over a wide range of model assumptions (Cosler et al., 2007). A cost-minimization analysis has shown that first-cycle use of pegfilgrastim may be costneutral in patients in whom the predicted risk of FN is less than $20 \%$. These findings have important implications for clinical guidelines for preventing chemotherapyinduced neutropenia and FN (Rader, 2006; Althaus , 2007). The mean direct and indirect costs are usually three to tenfold and 1.5- to threefold greater for inpatients, respectively. Factors associated with higher direct costs of care include diagnosis and inpatient care; higher indirect costs, male versus female gender; higher total costs, a diagnosis of lymphoma and inpatient care. Indirect costs appear to account for as much as half of the total supportive care costs when febrile neutropenia is managed in the outpatient setting and about one fifth of the total supportive care costs in the inpatient setting (Bennett et al., 2007).

In this study, LOS was evaluated as an outcome of interest as a substantial proportion of the health care costs incurred by patients with cancer can be attributed directly or indirectly to the treatment of FN. Longer periods of hospitalization increase cost and negatively affect the quality of life of affected patients. The age group, cancer type, associated complications, severity of neutropenia and abnormal creatinine were significantly associated with longer LOS (greater than 5 days) and mortality. To the best of our knowledge, this association of vital instability and severity of neutropenia and abnormal creatinine has not been reported previously among patients with FN in our part of the world.

Vital instability, severity of dehydration and bacteremias were significantly associated with both longer LOS and mortality. Despite the widespread use and availability of powerful antibiotics, bacteremia remains the most important independent prognostic marker for mortality. Several complication variables were associated with a longer length of stay and death. These findings are consistent with previous reports that co-morbid conditions are high risk factors in adult populations.

This study involved a prospective analysis to see the impact of changes in vital signs, hydration and severity of neutopenia on the LOS and mortality. The small sample size and single center study has several limitations as it cannot be generalized to the entire population of the country. This data set includes all patients admitted to our hospital unselected by any criteria other than febrile neutropenia and cancer. Additional information regarding socioeconomic status and delay in treatment could be possible if sample size, duration of study and number of centers is increased. Despite the widespread approach of treating all FN neutropenic patients with empiric broadspectrum antibiotics for potential bacteremia, FN with cancer remains associated with a significant, mortality rate. This suggests that certain host or disease-related factors may play a role in influencing mortality resulting from $\mathrm{FN}$.

The results of this study indicate that age, vital instability, dehydration, neutropenia, high creatinine, culture positivity and hematological malignancy should be considered high risk factors in chemotherapy induced FN cancer patients. The identification of risk factors for poor outcomes may help in devising protocols for modified dosage or including GCFs initially. This may help reduce the cost of cancer care as well as reduce mortality and morbidity. Prospective studies of FN among chemotherapy induced cancer patients in multiple centers in Pakistan may be beneficial in evaluating these risk factors further.

\section{References}

Althaus BL (2007). Myeloid growth factor therapy for prophylaxis of febrile neutropenia in non-myeloid malignancies: appropriate doses and schedules. J Natl Compr Canc Netw, 5, 229-34.

Basu SK, Fernandez ID, Fisher SG, Asselin BL, Lyman GH (2005). Length of stay and mortality associated with febrile neutropenia among children with cancer. J Clin Oncol, 23, 7958-66.

Balducci L, Lyman GH (2001). Patients aged 70 are at high risk for neutropenic infection and should receive hemopoietic growth factors when treated with moderately toxic chemotherapy. J Clin Oncol, 19, 1583-5.

Bennett CL, Calhoun EA (2007). Evaluating the total costs of chemotherapy-induced febrile neutropenia: results from a pilot study with community oncology cancer patients. Oncologist, 12, 478-83.

Bennett CL, Schumock GT (2003). Cost analyses of adjunct colony stimulating factors for older patients with acute myeloid leukaemia: Can they improve clinical decision making? Drugs Aging, 20, 479-83.

Bhurgri Y (2004). Karachi cancer registry data - Implications in NCCP Pakistan. Asian Pac J Cancer Prev, 5, 86-91.

Caggiano V, Weiss RV, Rickert TS, et al (2005). Incidence, cost, and mortality of neutropenia hospitalization associated with chemotherapy. Cancer, 103, 1916-24.

Carbonero RC, Mayordomo JI, Tornamira MV, et al (2001). Granulocyte colony-stimulating factor in the treatment of high-risk febrile neutropenia: a Multicenter Randomized Trial. J Natl Cancer Inst, 93, 31-8.

Chrischilles E, Delgado DJ, Stolshek BS, et al (2002). Impact of age and colony-stimulating factor use on hospital length of stay for febrile neutropenia in CHOP-treated nonHodgkin's lymphoma. Cancer Control, 9, 203-11.

Cosler LE, Eldar-Lissai A, Culakova E, Kuderer NM, Dale D (2007). Therapeutic use of granulocyte colony-stimulating factors for established febrile neutropenia: effect on costs from a hospital perpective. Pharmacoeconomics, 25, 34351.

Hughes WT, Armstrong D, Bodey GP, et al (2002). 2002 Guidelines for the use of antimicrobial agents in neutropenic patients with cancer. Clin Infect Dis, 34, 730-51.

Khan MA, Siddiqui BK, Shamim A, et al (2004). Emerging bacterial resistance patterns in febrile neutropenic patients: experience at a tertiary care hospital in Pakistan. J Pak Med Assoc, 54, 357-60.

Klastersky J, Paesmans M, Rubenstein EJ, et al (2000). The Multinational Association for Supportive Care in Cancer Risk Index: A multinational scoring system for identifying low-risk febrile neutropenic cancer patients. J Clin Oncol, 18, 3038-51.

Kuerer NM, Dale DC, Crawford J, Cosler LE, Lyman GH (2006). Mortality, morbidity, and cost associated with febrile 
neutropenia in adult cancer patients. Cancer, 106, 2258-66.

Liou SY, Stephens JM, Carpiuc KT, Feng W (2007), Economic burden of haematological adverse effects in cancer patients : a systematic review. Clin Drug Invest, 27, 381-96.

Lyman GH, Dale DC, Crawford J (2003). Incidence and predictors of low dose-intensity in adjuvant breast cancer chemotherapy: A nationwide study of community practices. $J$ Clin Oncol, 21, 4524-31.

Lyman GH (2005). Guidelines of the National Comprehensive Cancer Network on the use of myeloid growth factors with cancer chemotherapy: A review of the evidence. $J$ Natl Compr Canc Netw, 3, 557-71.

Malik IA, Khan WA, Karim M, Aziz Khan MA (1995). Feasibility of outpatient management of fever in cancer patients with low-risk neutropenia: results of a prospective randomized trial. Am J Med, 98, 224-31.

Morrison VA, Picozzi V, Scott S, et al (2001). The impact of age on delivered dose intensity and hospitalizations for febrile neutropenia in patients with intermediate-grade nonHodgkin's lymphoma receiving initial CHOP chemotherapy: A risk factor analysis. Clin Lymphoma, 2, 47-56.

Ozer H, Armitage JO, Bennett CL, et al (2000). 2000 update of recommendations for the use of hematopoietic colonystimulating factors: Evidence-based, clinical practice guidelines - American Society of Clinical Oncology Growth Factors Expert Panel. J Clin Oncol, 18, 3558-85.

Ozer H (2006). The timing of chemotherapy-induced neutropenia and its clinical and economic impact. Oncology, 20 (5 Suppl 4), 11-5.

Rader M (2006). Granulocyte colony-stimulating factor use in patients with chemotherapy-induced neutropenia: clinical and economic benefits. Oncology, 20(5 Suppl 4), 16-21.

Ray-Coquard I, Borg C, Bachelot T, Fayette J, Zufferey L (2006), Prognostic factors for febrile neutropenia. Bull Cancer, 93, 501-6.

Repetto L, Biganzoli L, Koehne CH, et al (2003). EORTC Cancer in the Elderly Task Force guidelines for the use of colonystimulating factors in elderly patients with cancer. Eur $J$ Cancer, 39, 2264-72.

Shamsi TS, Irfan M, Ansari SH, et al (2004) Allogeneic peripheral blood stem cell transplantation in patients with haematological malignancies. J Coll Physicians Surg Pak, 14, 522-6.

Talcott JA, Finberg R, Mayer RJ, Goldman L (1988). The medical course of cancer patients with fever and neutropenia. Arch Intern Med, 148, 2561-8.

Timmer-Bonte JN, Adang EM, Smit HJ, Biesma B, Wilschut FA (2006). Cost-effectiveness of adding granulocyte colonystimulating factor to primary prophylaxis with antibiotics in small-cell lung cancer. J Clin Oncol, 24, 2975-7.

Vincenzino JV (1995) Health care costs: Market forces and reform. Oncology, 9, 367-368; 371- 74.

Weber RJ (1993). Pharmacoeconomic issues in the use of granulocyte-macrophage colony-stimulating factor for bone marrow transplantation or chemotherapy-induced neutropenia. Clin Ther, 15, 180-91. 